\title{
Melatonin for Sleep in Children with Autism: A Controlled Trial Examining Dose, Tolerability, and Outcomes
}

\author{
Beth A. Malow, MD, MS ${ }^{1}$, Karen W. Adkins, MA, RN ${ }^{1}$, Susan G. McGrew, MD², Lily Wang, \\ $\mathrm{PhD}^{3}$, Suzanne E. Goldman, $\mathrm{PhD}^{1}$, Diane Fawkes, $\mathbf{B S}^{1}$, and Courtney Burnette, $\mathbf{P h D}^{2}$ \\ ${ }^{1}$ Sleep Disorders Division, Department of Neurology and Kennedy Center, Vanderbilt University \\ School of Medicine, Nashville, Tennessee 37232 \\ ${ }^{2}$ Department of Pediatrics, Monroe Carell Children's Hospital at Vanderbilt, Nashville, Tennessee \\ 37232 \\ ${ }^{3}$ Department of Biostatistics, Vanderbilt University School of Medicine, Nashville, Tennessee \\ 37232
}

\begin{abstract}
Supplemental melatonin has shown promise in treating sleep onset insomnia in children with autism spectrum disorders (ASD). Twenty-four children, free of psychotropic medications, completed an open-label dose-escalation study to assess dose-response, tolerability, safety, feasibility of collecting actigraphy data, and ability of outcome measures to detect change during a 14-week intervention. Supplemental melatonin improved sleep latency, as measured by actigraphy, in most children at 1 or $3 \mathrm{mg}$ dosages. It was effective in week 1 of treatment, maintained effects over several months, was well tolerated and safe, and showed improvement in sleep, behavior, and parenting stress. Our findings contribute to the growing literature on supplemental melatonin for insomnia in ASD and inform planning for a large randomized trial in this population.
\end{abstract}

\section{Keywords}

melatonin; insomnia; actigraphy; clinical trial; Children's Sleep Habits Questionnaire; Child Behavior Checklist; Autism Diagnostic Observation Schedule

\begin{abstract}
Sleep difficulties, particularly insomnia, occur in 50-80\% of children with autism spectrum disorders (Couturier et al 2005; Krakowiak et al 2008; Souders et al 2009; Goldman et al 2011b) and are often accompanied by child and family distress (reviewed in Richdale and Schreck, 2009; and Hollway and Aman 2011). Disordered sleep may exacerbate core and related symptoms of autism including social interactions, repetitive behaviors, affective problems, and inattention/hyperactivity (Schreck et al 2004; Gabriels et al 2005; Malow et al 2006; Goldman et al 2009; Goldman et al 2011a). Therefore, interventions that target sleep may not only improve child health and child and family distress, but may also ameliorate core and related symptoms of autism.
\end{abstract}

\footnotetext{
Corresponding author: Beth Malow, MD, MS, Sleep Disorders Division, Department of Neurology and Kennedy Center, $116121^{\text {st }}$ Avenue South, Room Room A-0116, Vanderbilt University School of Medicine, Nashville, Tennessee 37232, Phone: 615-322-0283, Fax: 615-936-0223, beth.malow@vanderbilt.edu. 
Supplemental melatonin has a favorable side-effect profile and is inexpensive. Along with other complementary and alternative therapies, it has gained widespread acceptance by parents of children with ASD as an alternative to FDA-approved medications (Harrington et al 2006). Three recent reviews have been published on the use of melatonin for insomnia in children with ASD (Doyen et al 2011; Rossignol et al 2011; Guénolé F et al 2011). These reviews summarized the limitations of the existing literature, which includes small sample sizes (majority of studies containing 20 subjects or fewer), a mix of ASD with other neurodevelopmental disorders, limited controlled trials, and limited studies using objective outcome measures or examining dose-response or tolerability in a systematic fashion. The reviews concluded that while supplemental melatonin appears safe, well tolerated, and promising in terms of efficacy, its use in ASD is not yet evidence-based.

To address the limitations of prior trials, we carried out a pilot open-label study of supplemental melatonin. The primary objective of the study was to evaluate the possible therapeutic effectiveness of melatonin. If effective, we wanted to also (1) Determine which doses were effective, (2) Assess how quickly effective doses improve sleep, (3) Collect safety and tolerability data in a systematic fashion, (4) Define the feasibility of actigraphy data as an outcome measure, and (5) Assess the ability of questionnaire data to detect change with a 14-week intervention in this population. Our findings presented here will allow for planning of larger randomized multicenter trials of supplemental melatonin for insomnia in ASD.

\section{Methods \\ Participants}

This study was approved by our Institutional Review Board. The principal investigator holds an approved FDA Investigational New Drug Application (\#76105) to use supplemental melatonin (Natrol $\left.{ }^{\circledR}\right)$ for insomnia in ASD.

From subspecialty clinics, as well as from the community (e.g., local autism society, public schools), using flyers given to potential participants, accompanied by letters and emails to referring practitioners, we recruited children ages 3-10 years with a clinical diagnosis of an autism spectrum disorder (autism, pervasive developmental disorder, not otherwise specified, or Asperger's disorder) whose parents reported sleep onset delay of 30 minutes of longer on three or more nights per week. A study coordinator was responsible for screening all potential participants to ensure that the above criteria were met, and consulted with the physician investigators as needed. Parents of these children provided informed consent and were enrolled in the protocol to begin study procedures. Children were free of psychotropic medications; allergy medications and medications for constipation were allowed. Parents agreed to avoid changes in current medications or the start of new medications during the time of study participation. Children with fragile X syndrome, Down syndrome, neurofibromatosis, or tuberous sclerosis complex and children who had a non-febrile unprovoked epileptic seizure within the last two years were excluded.

After enrollment, all children received:

1. Verification of the clinical diagnosis of ASD using a clinical interview that incorporated DSM-IV-TR criteria (American Psychiatric Association 2000) and the Autism Diagnostic Observation Schedule (ADOS; Lord 2000). A clinical psychologist with expertise in ASD diagnosis and who is research reliable on the ADOS administered these instruments to confirm participants' diagnoses.

2. A medical history, physical and Tanner staging by one of the authors, a pediatrician with expertise in ASD. Because of the effect of puberty on sleep and the unknown 
effects of melatonin on puberty, only children who were prepubertal continued in the study (excluded Tanner II or higher stage of physical development on medical examination or those with hormonal values for ACTH, cortisol, estrogen, testosterone, FSH, LH, and prolactin that were not consistent with prepubertal status). Children were also evaluated for comorbidities that affect sleep, including gastroesophageal reflux disease and psychiatric disorders. If these comorbidities were clinically determined to affect sleep, they were addressed prior to beginning melatonin. Children were excluded if laboratory values for blood count (CBC with platelets) and metabolic panel, including liver and renal function, were outside of the normal range.

3. A comprehensive sleep history of all children was performed by the principal investigator. Children suspected of having sleep apnea were evaluated with polysomnography prior to enrollment and excluded if sleep apnea was diagnosed.

\section{Study design}

As illustrated in Figure 1, a one-week baseline and two-week acclimation phase preceded the administration of supplemental melatonin. During the one-week baseline phase, parents received one hour of structured sleep education by the principal investigator, including establishment of a regular bedtime and wake time. Parents also received education in actigraphy procedures (see below). Children wore actigraphy watches to confirm that sleep latency (time to fall asleep) was at least 30 minutes on three or more nights in the week. During the two-week acclimation phase, parents gave their children an inert liquid 30 minutes before bedtime that was flavored similar to supplemental melatonin (compounded by Pharmacare, Mt. Juliet, TN®), in order to acclimate the child to taking a liquid medication before bedtime. Children were then given liquid supplemental melatonin (Natrol®, Chatsworth CA) 30 minutes before bedtime according to an optional escalating dose protocol based on three-week periods (Figure 1). The rationale for this design was to determine the lowest possible dose that was effective and well tolerated. The child was initially given $1 \mathrm{mg}(4 \mathrm{ml})$ melatonin for 3 weeks. If a satisfactory response occurred, defined as falling asleep within 30 minutes in five or more nights/week (for at least one of the weeks) as documented by actigraphy, melatonin was continued at its current dose until the end of the 14 week dosing period. If a satisfactory response did not occur at the $1 \mathrm{mg}$ dose, melatonin was increased to $3 \mathrm{mg}$ for three weeks. If a satisfactory response did not occur at the $3 \mathrm{mg}$ dose, melatonin was increased to $6 \mathrm{mg}$ for three weeks. If a satisfactory response did not occur at the $6 \mathrm{mg}$ dose, melatonin was increased to $9 \mathrm{mg}$ for three weeks. In the last two weeks of the dosing period, the child remained on the dose at which the satisfactory response occurred.

\section{Monitoring for adverse effects}

Parents were asked to review the Hague Side Effects Scale (Carpay et al 1996) each week throughout the 14-week dosing period. They were called at the end of each week by the study coordinator, and during the call, responses on the scale was reviewed with them.

\section{Outcome measures}

Actigraphy-All children wore the AW-64 Actiwatch ${ }^{\circledR}$ device (Phillips Respironics, Bend, OR) during the 17-week protocol (1 week of baseline, 2 weeks of acclimation, and 14 weeks of melatonin dosing; Figure 1). Each device contains an accelerometer, which detects motion and translates it into an electrical signal, stored in memory within the devices as actigraphy counts. The devices were configured using a one-minute epoch with medium threshold and the validated software (Phillips Respironics, 2010) algorithm was used to 
estimate sleep parameters, based on thresholds for wake and sleep, as described in prior work (Kushida, et al 2001; Lichstein et al 2006; Mezick, et al 2009).

During the training session, the parent and child were introduced to the actigraphy device for placement on the non-dominant wrist. Parents were given a quiz to test their knowledge of the actigraphy device. The parent completed a daily sleep diary throughout the 17- week protocol to assist in interpretation of actigraphy data and was also asked to use the event marker present on the device to mark lights out (the time that the child first attempted to fall asleep). Children who had difficulty tolerating the device on the wrist were allowed to use an alternate validated method which consisted of placing the device on a non-dominant shoulder location (Souders et al 2009; Adkins et al, in press).

Parent-completed survey forms-Parents completed a battery of surveys to determine the ability of questionnaires to detect change with intervention. The battery was completed at the beginning of the study and a second time at the conclusion of the study intervention procedures. These survey forms included the Children's Sleep Habits Questionnaire (CSHQ), the Child Behavior Checklist (CBCL), The Repetitive Behavior Scale-Revised (RBS-R), and the Parenting Stress Index Short Form (PSI-SF).

The CSHQ (Owens 2000) was included as a parent-reported measure of sleep to complement the objective measurement obtained by actigraphy. We hypothesized that the CSHQ insomnia-related domains would be more likely to improve with melatonin than the non-insomnia domains (e.g., sleep related breathing, parasomnias). The CSHQ was initially validated in ages 4-10 years (Owens 2000) and subsequently validated in younger ages (Goodlin-Jones et al 2008). A higher score indicates more difficulty with sleep.

The CBCL (Achenbach 2001a, 2001b) was included as a parent-reported measure of daytime behavior. Because separate CBCL forms spanned the age range of our participants (one for ages $1 \frac{1 / 2}{2}$ to 5 years and one for ages 6-18 years), we included scales common to both forms which we believed might improve after improvement in sleep with supplemental melatonin, based on the available literature (Hollway and Aman 2011). These included the syndrome scales of anxious/depressed, withdrawn, attention problems, and aggressive behavior, and the Diagnostic and Statistical Manual (DSM) scales of affective problems, attention-deficit hyperactivity, and oppositional defiant disorder. The CBCL contains modules for ages 2-5 years (Achenbach 2001a) and 6-18 years (Achenbach 2001b). A higher score indicates more difficulty with behavior.

The RBS-R (Bodfish 2000) was included as a parent-reported measure of repetitive behavior and restricted interests that include the following behavioral subscales: stereotyped, selfinjurious, compulsive, ritualistic, sameness, and restricted. The RBS-R has been validated in children (Lam and Aman 2007; Mirenda et al 2010). A higher score indicates more difficulty with behavior.

The PSI-SF (Abidin 1995) was included as a parent-reported measure of parenting stress that yields a total stress score from three scales: Parental Distress, Parent-Child Dysfunctional Interaction, and Difficult Child. It has been validated in children younger than 12 years. A higher score indicates higher levels of parent stress.

Parents were also asked to complete information about their education and occupation to provide estimates of socioeconomic status based on the Hollingshead Four Factor Index of Social Status (Hollingshead 1975). 


\section{Additional assessments}

The Peabody Picture Vocabulary Test- III (PPVT-III; Dunn 1997) and The Kaufman Brief Intelligence Test- Second Edition (K-BIT-2; Kaufman 2004) were used to characterize the receptive language skills and verbal and non-verbal cognitive functioning of our sample. Since the KBIT-2 was designed for children four years of age and older, it was not used for participants below this age group.

\section{Data Analysis}

Data were analyzed using SAS statistical software (version 9.1, SAS Institute Inc., Cary, NC) and SPSS statistical software (version 19, SPSS Inc., Chicago, IL). Given that this is a pilot study, in Table 2 and Table 3, we present means, standard deviations, and uncorrected p-values for all parameters analyzed. However, to assist with selecting the most robust measures for a larger controlled trial, we interpreted our data conservatively by controlling family wise error rate for the multiple measures. More specifically, as suggested in Westfall et al. (1999), we defined a family of tests to be all the tests that formed a natural and coherent unit (e.g. all items in a subscale of a questionnaire, or all the sleep parameters), and a more stringent threshold for p-values (i.e. $0.05 /$ number of tests in the family) was used to determine statistical significance.

Actigraphy-For each participant, average sleep parameters were computed at each phase: baseline, acclimation dosing phase, satisfactory dosing phase, and end of study dosing phase. Within group comparison of the sleep parameters at different phases were then analyzed with the Wilcoxon signed-rank test. This nonparametric test was used because the outcomes measured do not necessarily follow the normal distribution. Our major outcome variable was sleep latency. We also examined, as secondary outcome variables, total sleep time and sleep efficiency (total sleep time/time in bed), and wake time after sleep onset. In Table 2, since there were a total of 8 tests conducted for the set of sleep parameters, we considered p-values of less than $0.05 / 8$ (or 0.006) to be significant.

Parent-completed survey forms-For each participant, we compared the pre- and posttreatment variables for each scale using the Wilcoxon signed rank test. For the CSHQ (nine comparisons), p-values of less than 0.0056 were considered significant. Similarly, for the CBCL (seven comparisons), significance level was set at 0.0071, for the RBS (six comparisons), significance level was set at 0.0083 , and for the PSI (three comparisons), significance was set at 0.017 .

\section{Results}

Participants

Forty-six participants were enrolled in the study, and 24 completed all study procedures. Of the 22 children who were enrolled but did not complete the study, 20 were not started on melatonin for the following reasons-- Eight children did not have confirmatory diagnoses of ASD, six parents withdrew because the protocol was too time-consuming, two were lost to follow-up, one child improved after parent sleep education, falling asleep within 30 minutes on most nights (based on actigraphy), one child started medications, one child was Tanner stage 2, and one child had elevated liver enzymes. Two children were started on melatonin but did not complete the study. One child experienced a generalized tonic-clonic seizure. The child had no previous history of seizures, although as part of a separate research protocol, an EEG-polysomnogram done prior to the initiation of melatonin showed evidence for an epileptic seizure and interictal epileptiform activity. Melatonin was discontinued and the child was withdrawn from the study. Our medical safety monitor and Institutional Review Board reviewed this adverse event and determined it was not related to melatonin 
treatment. A second child, who was subsequently diagnosed with bipolar disorder, was unable to tolerate wrist actigraphy (the alternative actigraphy placement method was not developed at the time of her testing) and did not complete the study. She was given melatonin according to study protocol up to $3 \mathrm{mg}$, and based on parent diary, the child's sleep did not improve with melatonin treatment. The higher melatonin doses were not given based on the child's lack of improvement with lower doses, and the child's medical status was reevaluated. She was diagnosed with bipolar disorder, begun on respiridone, and both behavioral symptoms and sleep onset insomnia improved with treatment. The parent did not complete post-intervention surveys; however, clinical follow-up at one year indicated maintenance of the improvement in sleep and psychiatric symptoms.

\section{Actigraphy}

All children who completed the study tolerated actigraphy for the entire 17-week monitoring period, with five requiring an alternative placement. There were no significant differences in sleep parameters between the baseline and acclimation phase. Table 2 shows the sleep parameters by study phase, and Figure 1 illustrates the change in sleep latency with melatonin treatment, which decreased significantly with treatment $(\mathrm{p}<0.0001)$. The improvement in sleep latency was maintained until the end of the study. Analysis of sleep latency within the $1 \mathrm{mg}$ and $3 \mathrm{mg}$ dosing periods showed that the satisfactory response was achieved within the first week of treatment (i.e., the second and third week sleep parameters were not significantly different from the first week). Sleep duration, wake time after sleep onset, and sleep efficiency were not significantly different with melatonin treatment.

\section{Dose-response}

All 24 children who completed study procedures obtained a satisfactory response (as defined above) to melatonin at doses between $1 \mathrm{mg}$ and $6 \mathrm{mg}$. Seven children obtained a satisfactory response at $1 \mathrm{mg}, 14$ at $3 \mathrm{mg}$, and only 3 required $6 \mathrm{mg}$. The child's age or weight was not associated with melatonin dose response. The mean age/weight (standard deviation) of children responding to $1 \mathrm{mg}$ was $5.9(1.9)$ years/26.4 (11.1) $\mathrm{kg}$; and to 3 or $6 \mathrm{mg}$ was 5.9 (2.3) years/25.4 (11.2) kg.

\section{Questionnaires (Table 3)}

On the Children's Sleep Habits Questionnaire (CSHQ), a parent-based measure of sleep difficulties, the sleep onset delay, sleep duration, and sleep total subscales improved significantly after treatment with melatonin. Of note, several subscales (e.g. parasomnia, sleep disordered breathing) that would not be expected to improve with melatonin treatment did not improve, suggesting that parents were not answering indiscriminately that sleep difficulties has improved.

On the Child Behavior Checklist (CBCL), the withdrawn, affective, and ADHD subscales improved significantly after treatment with melatonin.

On the Repetitive Behavior Scale (RBS), the stereotyped and compulsive subscales improved significantly after treatment with melatonin.

On the Parenting Stress Index (PSI), the difficult child subscale improved significantly after treatment with melatonin.

\section{Laboratory findings}

No alterations in laboratory findings $(\mathrm{CBC}$, metabolic profile including liver and renal function, $\mathrm{ACTH}$, cortisol, estrogen, testosterone, FSH, LH, or prolactin) were noted with treatment. 


\section{Adverse effects and tolerability}

Only one child exhibited possible mild adverse effects related to the melatonin preparation (loose stools). All other children tolerated melatonin without difficulty.

\section{Discussion}

In this open-label dose-escalation study of supplemental melatonin, we found that (1) The majority of children responded to a $1 \mathrm{mg}$ or $3 \mathrm{mg}$ dose given 30 minutes before bedtime with an improvement in sleep latency; (2) This improvement was seen within the first week of dosing at the effective dose; (3) The medication was tolerated well with minimal adverse effects and no changes in laboratory values; (4) Actigraphy data was collectable over 17 weeks; and (5) Actigraphy, as well as parent-completed surveys focusing on sleep and behavior, showed change with the intervention.

Our findings are unique in that our study design allowed us to identify the doses at which children responded to melatonin (defined as sleep latency of 30 minutes or less on five or more nights in the week) and to define the time course of responsiveness (e.g., how many weeks were needed to observe a response). These results are not only helpful in the clinical care of children with ASD but also in planning for future randomized clinical trials. Safety and tolerability were also addressed in a comprehensive fashion, with reference to a side effects scale and laboratory testing. In agreement with a retrospective review of 107 children with ASD (Andersen et al. 2007), side effects were minimal.

We also documented that actigraphy can be used successfully in a 17-week trial in ASD. To our knowledge, no prior studies of melatonin in ASD have used actigraphy in a trial lasting several months. Actigraphy provided an important outcome measure that was objective and complemented that of parent report. Its use of 17 weeks allowed us to identify a satisfactory dose and document that effects were maintained over several months. The use of an alternative placement allowed us to optimize data collection and include children who did not tolerate standard wrist actigraphy.

In agreement with prior studies, we documented an improvement in sleep latency with melatonin treatment. Because our study criteria were designed to enroll children with sleeponset delay, we cannot definitively comment on the effects of melatonin on sleep duration or night wakings. A meta-analysis of randomized double-blind placebo-controlled studies in ASD that reported quantitative data (5 studies, 57 participants total), comparing melatonin treatment with baseline (pre-melatonin treatment) and with placebo, showed improved sleep latency and improved sleep duration but not night wakings (Rossignol and Frye 2011). Our findings were consistent with prior reports in that CSHQ sleep duration improved significantly with melatonin treatment, but night wakings did not. Neither sleep duration or night wakings, as measured by actigraphy, showed significant improvement with melatonin treatment. Large randomized controlled trials using objective measures of sleep will be needed to definitely establish the impact of melatonin on sleep duration and night wakings. The design of such trials may take several forms, depending on the questions of interest. Given our findings showing that a satisfactory response in sleep latency occurred within one week of dosing, a randomized trial of melatonin (parallel or crossover design) with a treatment phase as short as one week is reasonable to document improvements in sleep-onset insomnia. Alternatively, if more longer term outcome measures besides sleep latency were of interest, such as parenting stress, a longer treatment phase (e.g., one month or longer) may be appropriate.

The behavioral outcome measures that showed change with melatonin (e.g., attention-deficit hyperactivity, withdrawn, affective problems, stereotyped behaviors, compulsive behaviors) 
resemble that of prior work. The literature emphasizes that the behavioral construct of hyperactivity is affected by sleep disturbance - this had been documented in ASD populations (Goldman et al 2009; Mayes and Calhoun 2009) as well as typically developing children treated for obstructive sleep apnea (Chervin et al 2006). Other behavioral parameters which have been associated with poor sleep in children with ASD include repetitive behavior, including compulsive behavior, and oppositional and aggressive behavior, anxiety, depression, and mood variability (Malow et al 2006; Goldman et al, 2009; Mayes and Calhoun 2009). In an intervention study of parent education, hyperactivity and restricted behaviors showed improvements with treatment (Reed 2009).

Parenting stress, as measured by the Difficult Child Subscale, improved with treatment. We did not find improvement in the PSI parent-related domains (Parental Distress or ParentChild Dysfunctional Interaction) suggesting that parental stress in autism is multifactorial and may not be addressed with a single intervention.

Although melatonin is safe and well tolerated, we believe that it should be administered under the treatment of a physician. This is because of the importance of assessing children with ASD and insomnia for medical, neurological, and psychiatric comorbidities, which may cause or contribute to insomnia. This point is illustrated by the one non-responder in our study, a child subsequently diagnosed with bipolar disorder.

Strengths of our design include: (1) Participants limited to those meeting clinical and research criteria for ASD; (2) Relatively large sample size compared to prior studies; (3) Standardized parent sleep education protocol administered prior to the treatment with melatonin; (4) Use of objective primary outcome measures (actigraphy); (5) Screening for medical comorbidities which can contribute to insomnia; (6) Assessment of effect of improved sleep on behavioral outcomes (e.g., ameliorating core and associated features of autism and family functioning); and (7). Of patients whose cognitive skills were evaluated, all had an IQ of 70 or higher on the verbal or non-verbal scales, or both. Thus, our population had ASD with normal intelligence, eliminating any concerns about the impact of intellectual disability. The lack of significant findings on some of the behavioral subscales may reflect our small sample size. Another limitation is that we did not include a placebo group; large randomized multicenter trials will need to include a placebo group to establish efficacy. While our children were free of psychotropic medications, which can be viewed as a relative strength, our results are less generalizable to the autism population with sleeponset delay, in which some children are taking medications (e.g., antidepressants and stimulants) which interfere with sleep or with hepatic enzymes (CYP1A2) that metabolize melatonin. Finally, we cannot comment on the dosing, safety, and tolerability of melatonin in children older than age 10 or who have entered puberty.

In summary, our findings provide unique information on dosing, tolerance/safety, and outcome measures for the use of melatonin in pre-pubertal children with ASD. They add to the growing literature documenting that melatonin shows promise for treating sleep-onset insomnia in ASD, and address key issues needed to design a large controlled trial of melatonin in this population.

\section{Acknowledgments}

Financial support: This work was supported by NICHD (RO1 HD59253), Autism Speaks, Vanderbilt General Clinical Research Center (M01 RR-00095 from the National Center for Research Resources, National Institutes of Health), and by the Vanderbilt University Kennedy Center (NICHD HD15052). Natrol ®, (Chatsworth, CA) provided study drug but no other support. Dr. Shlomo Shinnar provided valuable input into the study design. We acknowledge Ms. Kyla Surdyka and Ms. Meg Touvelle for their assistance with data entry, and are appreciative to the families who participated in this project. 


\section{References}

Abidin, RR. Parenting Stress Index. 3. Odessa, FL: Psychological Assessment Resources; 1995.

Adkins KW, Goldman SE, Fawkes D, Surdyka K, Wang L, Song Y, et al. A pilot study of shoulder placement for actigraphy in children. Behavioral Sleep Medicine. in press.

Achenbach, TM.; Rescorla, LA. Manual for the ASEBA Preschool Forms and Profiles. Burlington, VT: University of Vermont, Research Center for Children, Youth, and Families; 2001a.

Achenbach, TM.; Rescorla, LA. Manual for the ASEBA School Age Forms and Profiles. Burlington, VT: University of Vermont, Research Center for Children, Youth, and Families; 2001b.

American Psychiatric Association. Diagnostic and Statistical Manual of Mental Disorders. 4. Washington DC: American Psychiatric Association; 2000. Text Revision

Andersen IM, Kaczmarska J, McGrew SG, Malow BA. Melatonin for insomnia in children with autism spectrum disorders. Journal of Child Neurology. 2008; 23(5):482-5. [PubMed: 18182647]

Bodfish JW, Symons FJ, Parker DE, Lewis MH. Varieties of repetitive behavior in autism: comparisons to mental retardation. Journal of Autism \& Developmental Disorders. 2000; 30(3): 237-43. [PubMed: 11055459]

Carpay JA, Arts WFM, Vermeulen J, Stroink H, Brouwer OF, Peters ACB, et al. Parent-completed scales for measuring seizure severity and severity of side-effects on antiepileptic drugs in childhood epilepsy: development and psychometric analysis. Epilepsy Research. 1996; 24:173-81. [PubMed: 8832193]

Chervin RD, Ruzicka DL, Giordani BJ, et al. Sleep-disordered breathing, behavior, and cognition in children before and after adenotonsillectomy. Pediatrics. 2006; 117:769-778.

Couturier JL, Speechley KN, Steele M, Norman R, Stringer B, Nicholson R, et al. Parental perception of sleep problems in children of normal intelligence with pervasive developmental disorders: Prevalence, severity, and pattern. Journal of the American Academy of Child and Adolescent Psychiatry. 2005; 44:815-822. [PubMed: 16034284]

Doyen C, Mighiu D, Kaye K, Colineaux C, Beaumanior C, Mauraeff Y, et al. Melatonin in children with autistic spectrum disorders: Recent and practical data. European Child \& Adolescent Psychiatry. 2011; 20:231-239. [PubMed: 21359552]

Dunn, LM. Peabody Picture Vocabulary Test. 3. Minneapolis, MN: American Guidance Service; 1997.

Gabriels RL, Cuccaro ML, Hill DE, Ivers BJ, Goldson E. Repetitive behaviors in autism: relationships with associated clinical features. Res Developmental Disabilities. 2005; 26:169-181.

Goldman SE, Surdyka K, Cuevas R, Adkins K, Wang L, Malow BA, et al. Defining the sleep phenotype in children with autism. Developmental Neuropsychology. 2009; 34:560-573. [PubMed: 20183719]

Goldman SE, Mc Grew S, Johnson KP, Richdale AL, Clemons T, Malow BA. Sleep is associated with problem behaviors in children and adolescents with autism spectrum disorders. Research in Autism Spectrum Disorders. 2011a; 5:1223-1229.

Goldman SE, Richdale AL, Clemons T, Malow BA. Sleep behaviors in autism spectrum disorders Variations in age from early childhood through adolescence. Journal of Autism and Developmental Disorders. 2011b May 3. epub ahead of print.

Goodlin-Jones BL, Sitnick SL, Tang K. The Children's Sleep Habits Questionnaire in toddlers and preschool children. J Dev Behav Pediatr. 2008; 29:82-88. [PubMed: 18478627]

Guénolé F, Godbout R, Nicolas A, Franco P, Claustrat B, Baleyte JM. Melatonin for disordered sleep in individuals with autism spectrum disorders: Systematic review and discussion. Sleep Medicine Reviews. 2011; 15(6):379-387. [PubMed: 21393033]

Harrington JW, Rosen L, Garnecho A. Parental perceptions and use of complementary and alternative medicine practices for children with autism spectrum disorders in private practice. J Dev Behav Pediatr. 2006; 27(2 Suppl):S156-61. [PubMed: 16685182]

Hollway JA, Aman MG. Sleep correlates of pervasive developmental disorders: A review of the literature. Research in Developmental Disabilities. 201110.1016/j.ridd.2011.04.001

Hollingshead, AB. Four factor index of social status. New Haven, CT: Yale University, Department of Sociology; 1975. 
Johnston C, Mash EJ. A measure of parenting satisfaction and efficacy. J Clin Child Psychology. 1989; 17:167-175.

Kaufman, AS.; Kaufman, NL. Kaufman Brief Intelligence Test. 2. Circle Press, MN: American Guidance Service, Inc; 2004.

Krakowiak P, Goodlin-Jones B, Hertz-Picciotto I, Croen LA, Hansen RL. Sleep problems in children with autism spectrum disorders, developmental delays, and typical development: A populationbased study. J Sleep Res. 2008; 17:197-206. [PubMed: 18482108]

Kushida CA, Chang A, Gadkary C, Guilleminault C, Carrillo O, Dement WC, et al. Comparison of actigraphic, polysomnographic, and subjective assessment of sleep parameters in sleep-disordered patients. Sleep Med. 2001; 2:389-96. [PubMed: 14592388]

Lam KSL, Aman MG. The Repetitive Behavior Scale-Revised: independent validation in individuals with autism spectrum disorders. J Autism Dev Disord. 2007; 37(5):855-66. [PubMed: 17048092]

Lichstein KL, Stone KC, Donaldson J, Nau SD, Soeffing JP, Murray D, et al. Actigraphy validation with insomnia. SLEEP. 2006; 29:232-9. [PubMed: 16494091]

Lord C, Risi S, Lambrecht L, Cook EH Jr, Leventhal BL, DiLavore PC, et al. The autism diagnostic observation schedule-generic: a standard measure of social and communication deficits associated with the spectrum of autism. J Autism Dev Disord. 2000; 30(3):205-23. [PubMed: 11055457]

Malow BA, Marzec ML, McGrew SG, Wang L, Stone W. Characterizing sleep in children with autism spectrum disorders: A multidimensional approach. SLEEP. 2006; 29:1563-1571. [PubMed: 17252887]

Mayes SD, Calhoun S. Variables related to sleep problems in children with autism. Research in Autism Spectrum Disorder. 2009; 3:931-941.

Mezick EJ, Matthews KA, Hall M, Kamarck TW, Buysse DJ, Owens JF, et al. Intra-individual variability in sleep duration and fragmentation: Associations with stress. Psychoneuroendocrinology. 2009; 34:1346-1354. [PubMed: 19450933]

Mirenda R, Smith IM, Vaillancourt T. Validating the Repetitive Behavior Scale-Revised in young children with autism spectrum disorder. J Autism Dev Disord. 2010; 40:1521-1530. [PubMed: 20405194]

Owens JA, Spirito A, McGuinn M. The Children's Sleep Habits Questionnaire (CSHQ): psychometric properties of a survey instrument for school-aged children. SLEEP. 2000; 23:1043-1051. [PubMed: 11145319]

Phillips Respironics. Actiware / Actiware-ct. Software Version 5.9. 2010. Actiwatch communication and sleep analysis software instruction manual.

Richdale AL, Schreck KA. Sleep problems in autism spectrum disorders: prevalence, nature, \& possible biopsychosocial aetiologies. Sleep Med Rev. 2009; 13(6):403-11. [PubMed: 19398354]

Reed HE, McGrew SG, Artibee K, Surdyka K, Goldman SE, Frank K. Parent-based sleep education workshops in autism. Journal of Child Neurology. 2009; 24:236-245.

Rossignol D, Frye R. Melatonin in autism spectrum disorders: a systemic review and meta-analysis. Developmental Medicine \& Child Neurology. 2011; 53(9):783-792. [PubMed: 21518346]

Schreck KA, Mulick JA, Smith AF. Sleep problems as possible predictors of intensified symptoms of autism. Res Dev Disabil. 2004; 25:57-66. [PubMed: 14733976]

Souders MC, Mason TB, Valladares O, Bucan M, Levy SA, Mandell DS, et al. Sleep behaviors and sleep quality in children with autism spectrum disorders. SLEEP. 2009; 32:1566-1578. [PubMed: 20041592]

Westfall, P.; Tobias, RD.; Ron, D.; Wolfinger, RD.; Hochberg, Y. Multiple Comparisons and Multiple Tests Using SAS. Cary, NC: SAS Institute Inc; 1999. 


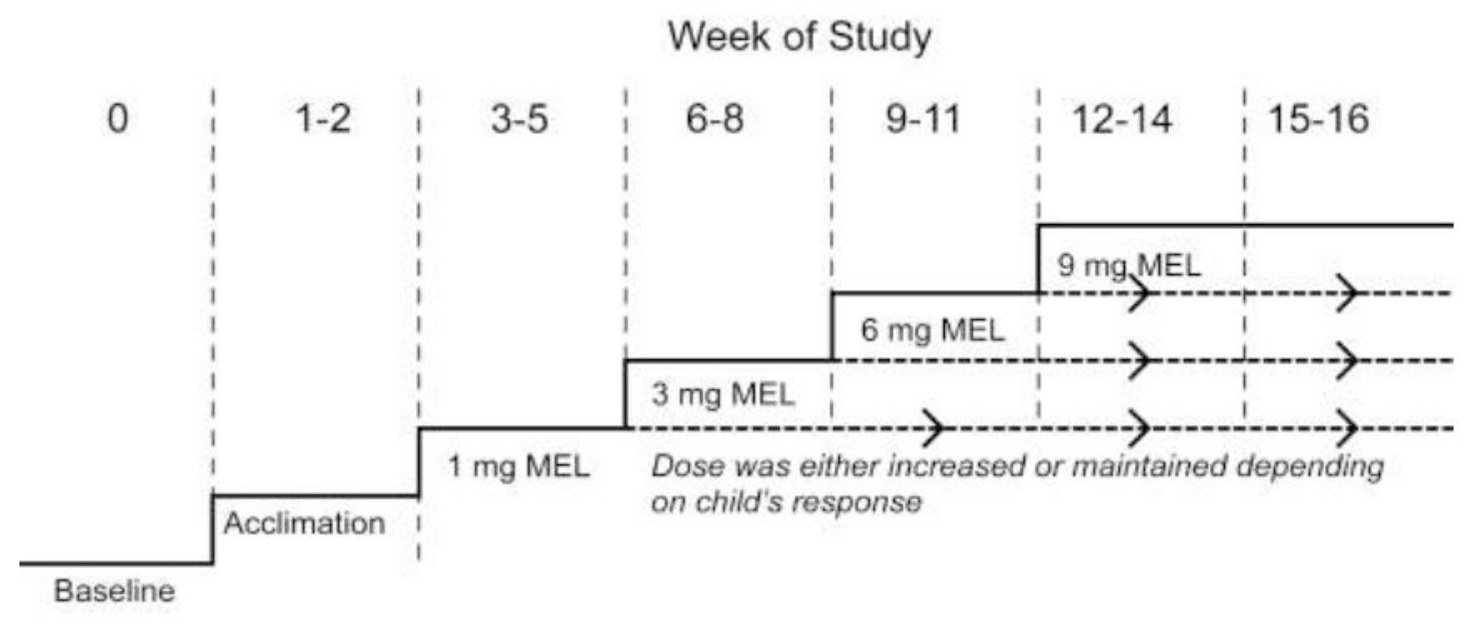

Figure 1. Optional Escalation Study Design

After the baseline and acclimation (inert liquid) phases, supplemental melatonin was increased if the child did not exhibit a satisfactory response, defined as a sleep latency of 30 minutes or less on five or more nights in any given week within the three week dosing period. In the last two weeks of the dosing period, the child remained on the dose at which the satisfactory response occurred. 


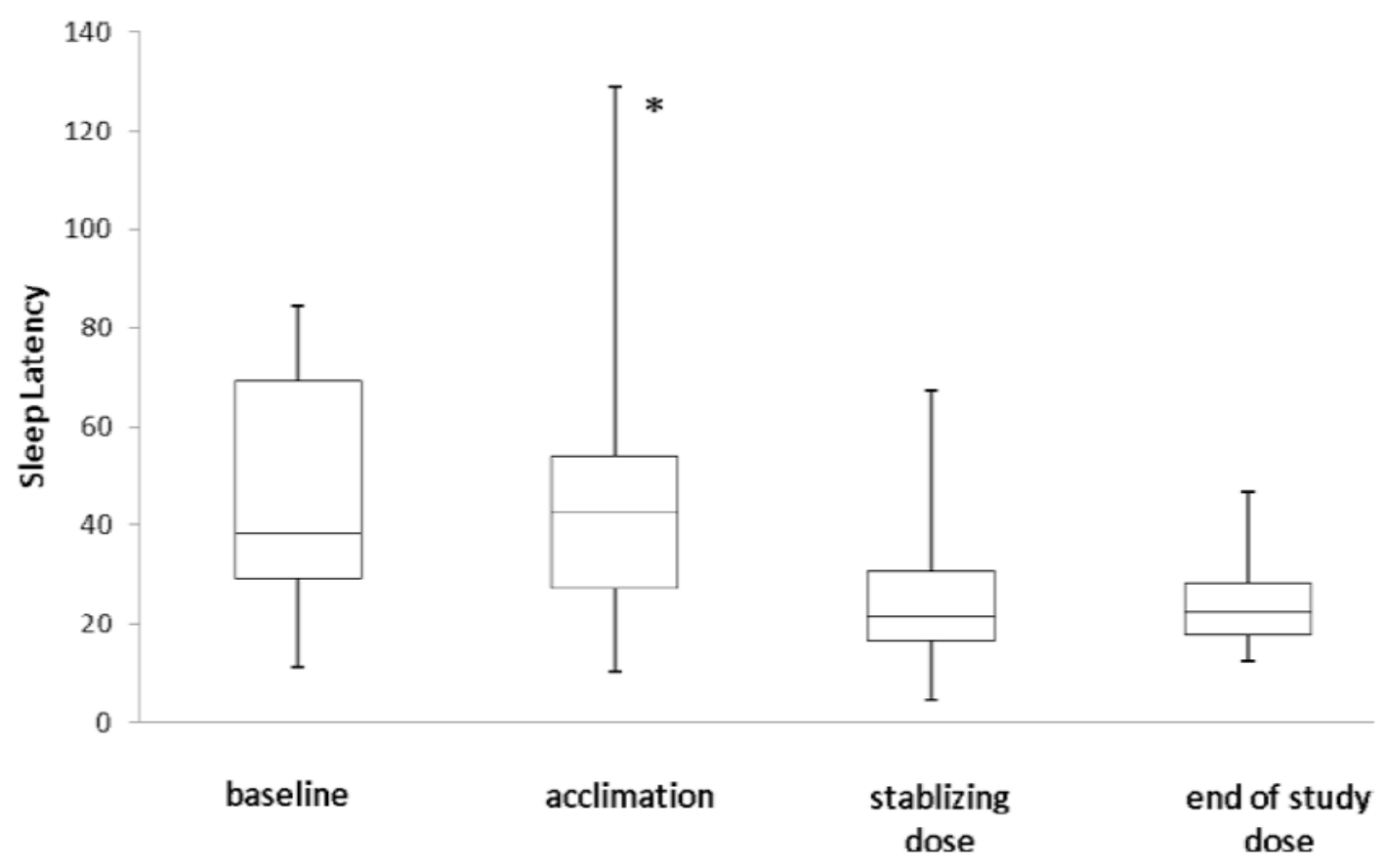

Figure 2. Change in Sleep Latency with Melatonin Treatment

Median sleep latency (y-axis) in minutes was measured by actigraphy at four different time points: (a) baseline phase; (b) acclimation phase (weeks 1-2) when child received inert liquid; (c) stabilizing dose phase, the first three-week period when child's sleep latency was less than 30 minutes for 5 or more nights in at least one of the weeks; and (d) end of study phase, the last two weeks of treatment. The lines in the box plot correspond to maximum, 3 rd quartile ( 75 th percentile), median, 1 st quartile (25th percentile) and minimum. The asterisk (*) indicates that the median sleep latency in the acclimation phase was significantly different than in the stabilizing dose or end of study phase $(\mathrm{p}<0.0001)$. 


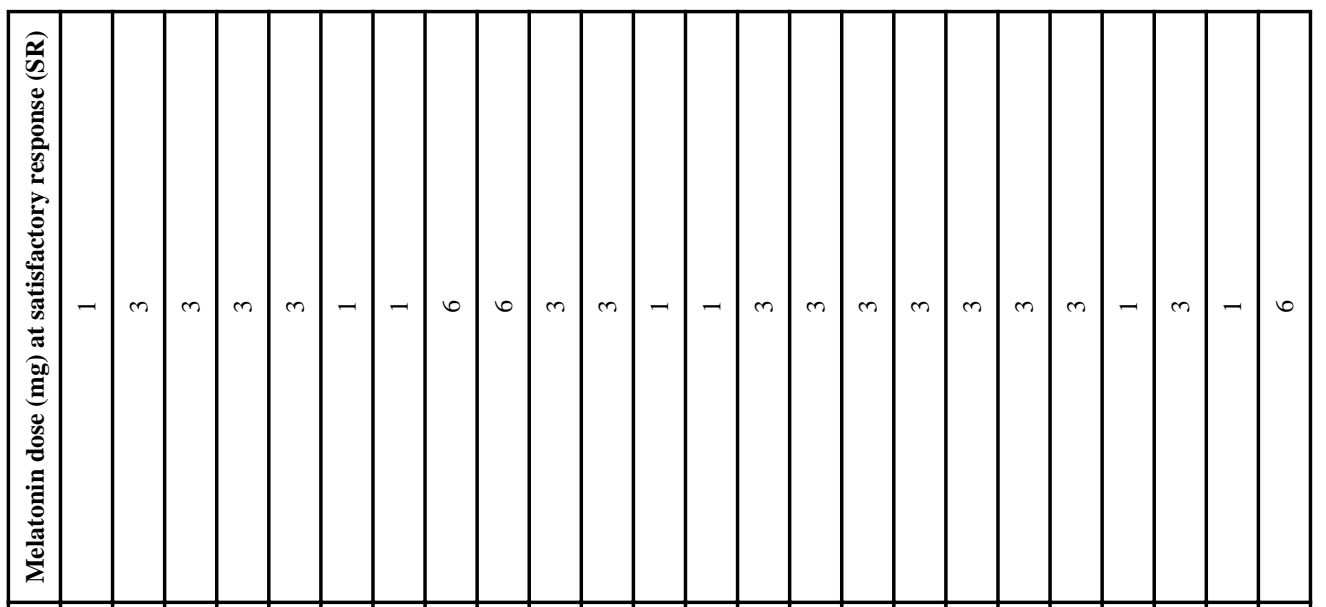




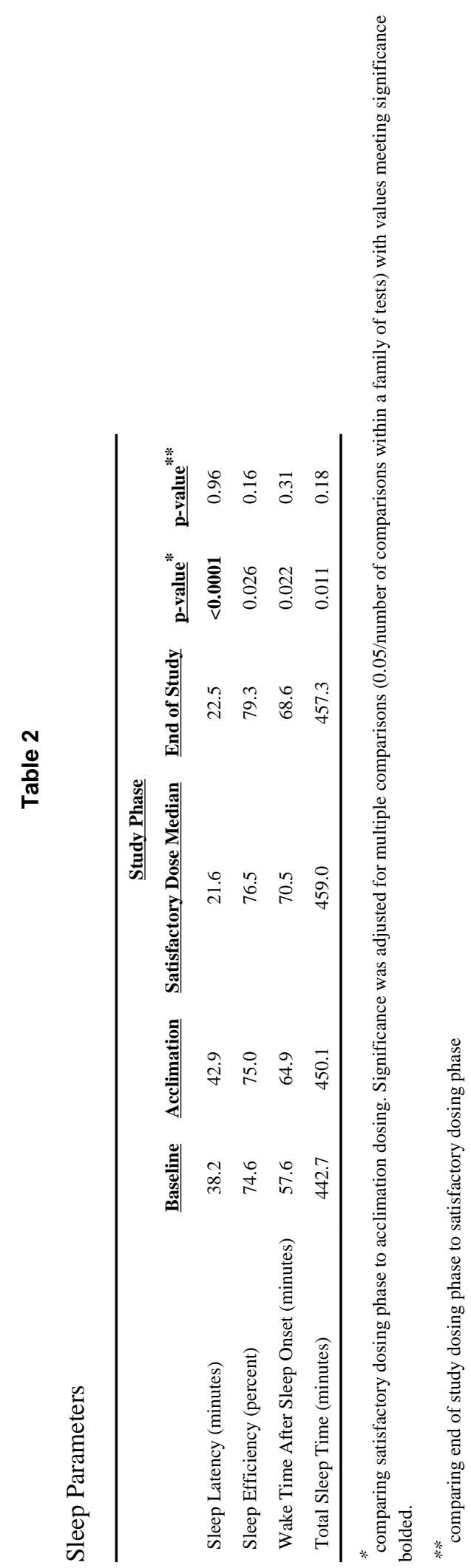

J Autism Dev Disord. Author manuscript; available in PMC 2013 August 01. 
Table 3

Parental Report Parameters

\begin{tabular}{|c|c|c|c|}
\hline & \multicolumn{2}{|c|}{ Study Phase } & \multirow[b]{2}{*}{ p-value } \\
\hline & Baseline & End of Study & \\
\hline & \multicolumn{2}{|c|}{ Mean (Standard Deviation) } & \\
\hline \multicolumn{4}{|l|}{ Children's Sleep Habits Questionnaire } \\
\hline Bedtime resistance & $10.7(4.2)$ & $8.3(2.3)$ & 0.008 \\
\hline Sleep onset delay & $2.6(0.6)$ & $1.3(0.6)$ & $<0.0001$ \\
\hline Sleep duration & $6.4(1.8)$ & $3.7(1.3)$ & $<0.0001$ \\
\hline Sleep anxiety & $6.8(1.9)$ & $6.3(1.7)$ & 0.270 \\
\hline Night wakings & $5.3(1.9)$ & $4.3(1.4)$ & 0.023 \\
\hline Parasomnias & $9.7(2.0)$ & $9.2(2.1)$ & 0.780 \\
\hline Sleep disordered breathing & $3.8(1.2)$ & $3.5(0.6)$ & 0.170 \\
\hline Daytime sleepiness & $14.1(2.4)$ & $12.6(2.7)$ & 0.129 \\
\hline Sleep Total & $55.2(6.9)$ & $45.1(4.7)$ & $<0.0001$ \\
\hline \multicolumn{4}{|l|}{ Child Behavior Checklist } \\
\hline Anxious/depressed & $59.1(9.3)$ & $57.6(7.8)$ & 0.129 \\
\hline Withdrawn & $71.5(9.3)$ & $66.0(7.8)$ & $<0.0001$ \\
\hline Attention Problems & $65.6(8.3)$ & $63.0(9.3)$ & 0.069 \\
\hline Aggressive Behavior & $62.3(11.5)$ & $60.0(9.4)$ & 0.073 \\
\hline Affective Problems & $69.2(7.1)$ & $60.8(6.5)$ & $<0.0001$ \\
\hline DSM Attention/Deficit Hyperactivity & $63.6(8.2)$ & $60.4(8.2)$ & 0.006 \\
\hline DSM Oppositional Behavior & $62(9.6)$ & $59(8.3)$ & 0.026 \\
\hline \multicolumn{4}{|l|}{ Repetitive Behavior Scale } \\
\hline Stereotyped & $6.6(3.5)$ & $5.2(3.1)$ & 0.008 \\
\hline Self-injurious & $2.6(2.9)$ & $2.1(203)$ & 0.325 \\
\hline Compulsive & $7.1(5.2)$ & $4.5(3.7)$ & $<0.0001$ \\
\hline Ritualistic & $7.5(4.5)$ & $5.5(3.7)$ & 0.013 \\
\hline Sameness & $10.0(6)$ & $7.6(6.4)$ & 0.017 \\
\hline Restricted & $5.3(3.4)$ & $4(2.6)$ & 0.130 \\
\hline \multicolumn{4}{|l|}{ Parenting Stress Index } \\
\hline Parental Distress & $32.4(11.1)$ & $30.3(8.2)$ & 0.204 \\
\hline Parent-Child Dysfunctional Interaction & $29.1(9.0)$ & $25.9(8.4)$ & 0.098 \\
\hline Difficult Child & $41.3(7.2)$ & $36.1(7.0)$ & 0.003 \\
\hline
\end{tabular}

comparing baseline phase to end of study phase. Significance was adjusted for multiple comparisons $(0.05 /$ number of comparisons within a family of tests) with values meeting significance bolded.

On these scales, higher values indicate more difficulties. 